\title{
Automated Detection of Parenchymal Changes of Ischemic Stroke in Non-Contrast Computer Tomography: A Fuzzy Approach
}

\author{
A. Davis-Ortiz ${ }^{\mathrm{a}}$, N. Gordillo-Castillo ${ }^{\mathrm{b}^{*}}$, E. Montseny-Masip ${ }^{\mathrm{a}}$, F.X. Aymerich ${ }^{\mathrm{a}, \mathrm{c}}, \mathrm{M}$. \\ López-Córdova $^{d}$, J. Mejía-Muñoz ${ }^{b}$, L. Ortega-Maynez ${ }^{b}$, B. Mederos-Madrazo \\ ${ }^{a}$ Automatic Control Department, Universitat Politècnica de Catalunya - Barcelona Tech, Spain \\ ${ }^{b}$ Department of Electrical and Computer Engineering, Universidad Autónoma de Ciudad Juárez, México \\ ${ }^{c}$ MR Unit (IDI), Department of Radiology, Hospital Universitari Vall d'Hebron, Universitat Autònoma de Barcelona, Spain \\ ${ }^{d}$ General Hospital 35 Mexican Institute of Social Security, México \\ ${ }^{e}$ Department of Physics and Mathematics, Universidad Autónoma de Ciudad Juárez, México
}

\begin{abstract}
The detection of ischemic changes is a primary task in the interpretation of brain Computer Tomography (CT) of patients suffering from neurological disorders. Although CT can easily show these lesions, their interpretation may be difficult when the lesion is not easily recognizable. The gold standard for the detection of acute stroke is highly variable and depends on the experience of physicians. This research proposes a new method of automatic detection of parenchymal changes of ischemic stroke in Non-Contrast CT. The method identifies non-pathological cases ( 94 cases, 40 training, 54 test) based on the analysis of cerebral symmetry. Parenchymal changes in cases with abnormalities ( 20 cases) are detected by means of a contralateral analysis of brain regions. In order to facilitate the evaluation of abnormal regions, nonpathological tissues in Hounsfield Units were characterized using fuzzy logic techniques. Cases of non-pathological and stroke patients were used to discard/confirm abnormality with a sensitivity (TPR) of $91 \%$ and specificity (SPC) of $100 \%$. Abnormal regions were evaluated and the presence of parenchymal changes was detected with a TPR of $96 \%$ and SPC of $100 \%$. The presence of parenchymal changes of ischemic stroke was detected by the identification of tissues using fuzzy logic techniques. Because of abnormal regions are identified, the expert can prioritize the examination to a previously delimited region, decreasing the diagnostic time. The identification of tissues allows a better visualization of the region to be evaluated, helping to discard or confirm a stroke.
\end{abstract}

Keywords: Ischemic stroke; Brain Tissue Segmentation; Fuzzy Logic

\footnotetext{
${ }^{*}$ Corresponding author. Tel.: +526566884841

E-mail address: nelly.gordillo@uacj.mx.
} 


\section{Introduction}

Cerebrovascular diseases according to the World Health Organization are the third leading cause of death and the leading cause of disability in industrialized countries. The term "stroke" is used to describe the clinical phenomenon which consists of a sudden onset of neurological symptoms due to a cerebrovascular disorder. In contrast, cerebral infarction describes a lethal ischemic phenomenon at tissue level that corresponds to $85 \%$ of the cases of stroke [1]. A stroke can be classified into two major groups depending on its nature: hemorrhagic and ischemic [2]. The average duration of non-lacunar stroke evolution is 10 hours. Ischemia can cause functional neurological damage or may present as a cerebral infarction, which causes irreversible neuronal structural damage. Each minute, 1.9 million neurons, 14 billion synapses, and $12 \mathrm{~km}$ (7.5 miles) of myelinated fibers are destroyed [3].

Because of its wide availability, low cost, fast execution and proven efficacy, Non-contrast Cerebral Computer Tomography (NCCT) is the first choice for neuroradiology examination of all patients presenting an acute focal neurological deficit [4]. CT helps to differentiate the type of stroke and to discard other diseases that may cause a vascular process similar to a stroke such as tumors, bleeding, metastasis, etc. [5-10]. It also reports the dimensions of an ischemic lesion and indirect signs such as cortical grooves asymmetry, structural displacements, density increase in the middle cerebral artery in the basal tract, or attenuation coefficients of the brain's parenchyma [11-14]. Initial changes in CT may be present within the first 3 hours after the onset of the stroke and this may, or may not, influence the selection of patients for thrombolytic therapy $[15,16]$. More than half of protocol violations are due to a failure to recognize the first signs of infarction on initial CT [17]. There is considerable discordance, even among the more experienced physicians, about the recognition and quantification of such early CT changes [18], since it is subject to observer variability due to many factors [6]. The human eye is able to differentiate a limited number of 20 gray tones, which means that the contrast resolution is limited to 4 Hounsfield Units (HU). The window width of $80 \mathrm{HU}$ gives a remarkable maximum change of 1-2 gray levels within the first 4 hours of ischemia [19]. Diffuse changes can hardly be distinguished in noisy areas due to low contrast brightness, bone artifacts and non-optimal scanning.

Usually the detection of early changes of ischemic stroke in CT is performed manually by a radiologist and sometimes with the use of simple software tools such as contrast modification. There are various CADe (Computer Assisted Detection) schemes that integrate mathematical models to help identify abnormalities that physicians may overlook and, therefore, the software improves the efficiency of disease detection. CADe systems for automatic detection of early ischemic changes still need further research and improvement [20]. The implementation of an algorithm based on computer vision techniques, as part of the development of a CADe, would allow the expert radiologist (and not expert) to perform a detailed search on specific areas of the brain where the algorithm has detected the possible presence of a stroke. This would accelerate the beginning of the treatment that should be up to 7.3 hours after symptom onset [21]. By obtaining a more timely diagnosis and its consequent treatment, a disability and even death of the patient can be avoided, thus reducing morbidity and mortality.

Few contributions have been made in the literature of CADe based on computer vision techniques for ischemic changes detection. In [22] brain abnormality was inferred from the histogram comparison of hemispheres. The method was only tested in chronic infarction cases where the stroke is easily detectable. Other methods also use histogram-based comparison, starting from a segmented region of interest (ROI) [13]. Because early density changes can hardly be seen, it can be difficult to decide where to establish the ROI. Depending on where the ROI is established, the stroke can be overlooked. The comparison of symmetric ROIs 
does not necessarily mean a strict comparison of the corresponding structures due to partial volume effects, cerebrospinal fluid or white matter may affect the attenuation coefficients of a region.

In [23], the data was interpolated to a single volume: a skull-stripping algorithm was used, then normalized using an atlas, and segmented into anatomical regions. The voxel densities in the lentiform nucleus and insula were compared to the contralateral side using statistical analysis for the detection of hypodensity. A method of post-processing improvement with comparison of distributions of attenuation coefficients was proposed in [24]. It was based on the analysis of voxels using normalization, segmentation and differentiation of the original $\mathrm{CT}$ and the resulting filtered image.

Other well-known methods of local contrast enhancement to visualize hidden structures in medical imaging are based on the adaptive equalization of the histogram $[25,26]$. A local window is considered for each individual pixel, and then a new intensity value is calculated based on the local histogram. The method does not change the overall appearance of the image, which is important for clinical reading since such variations usually distract the attention of radiologists. However, shadow-like artifacts appeared in fairly homogeneous regions and, in some cases, thin structures were attenuated. These disadvantages were reduced when the adaptive equalization of the histogram was performed $[27,28]$.

This wavelet-based algorithm [29] was proposed for simultaneous automatic visualization of the full range of dynamic contrast of CT. Interpretation times were significantly reduced. Post-processing in the wavelet domain was less susceptible to artifacts and perturbations, unlike the exact representation of adaptive histogram equalization techniques. The diagnostic accuracy, however, was insufficient compared to conventional window viewing. Wavelets were also used to detect strokes [19]. The perception of early changes was improved by eliminating noise and enhancing local contrast. The method improved the subtlest signs of hypodensity, which were often invisible in the standard CT scan. Data processing became more effective through the initial segmentation of brain tissue and the extraction of regions susceptible to density changes. The sensitivity of stroke diagnosis increased to $56.3 \%$ compared with $12.5 \%$ of the standard CT scan.

Semiautomatic algorithms have also been proposed. In [30] the radiologist defines de ROI by selecting an initial seed point (a voxel) that belongs to the structure of interest. It uses intensity-based region growth algorithms that exclude ventricles and hemorrhages. The damages are evaluated according to the seed selected by the radiologist; however, the technique is too slow since it takes 2 hours per study.

Recently, there have been diverse contributions to the literature of methods that seek to automate the ASPECTS protocol. In [31] an automated ASPECTS scoring method was developed as an alternative to manual ASPECTS score. Based on the brain density shift between contralateral brain areas, the method quantifies subtle early ischemic changes. The method matched with manual consensus scoring in $73 \%$ of the cases without bias or outliers, in contrast with individual observers. A new software, the e-ASPECTS, was proposed for the automatic detection of acute ischemic stroke. The method segments the regions using texture information and an atlas. It generates a score and marks the regions that contain the damage. It offers a direct score based on the image in order to indicate or discard thrombolytic therapy. The e-ASPECTS has been evaluated in [32] and in [33] concluding that it is non-inferior to the ASPECTS results scored by three neuroradiologists on NCCT. 
Because the identification of early ischemic changes in acute strokes is still very subjective, we must continue to focus on the development of diagnostic tools that improve the perception of subtle changes and that allow stroke-specialists to determine an early diagnosis with greater precision.

Brain tissue segmentation (BTS) plays a key role in the study of various abnormalities, brain development and evaluation of the progress of treatment [34]. Cerebrospinal fluid (CSF), white matter (WM) and gray matter (GM) are the basic tissue types in BTS. Subsections of the image with specific characteristics are labeled with the aim of achieving homogeneous partitions representing CSF, WM and GM. Several segmentation techniques have been proposed in the literature for BTS, mostly designed for their use in magnetic resonance imaging (MRI) [35-37]. In CT the contributions have been limited to the segmentation of pathologies and brain parenchyma excluding the separation of tissues [38-40].

The inherent difficulty in segmenting and quantifying the various brain tissues has increased the use of soft computing techniques in BTS. Fuzzy logic as a part of soft computing has the potential to combine human heuristics in computer-aided decision making, it opens the door to construction of better models of reality and involves exploitation of a tolerance for imprecision [41]. Fuzzy logic has been applied in all disciplines of medicine in some form of classification, detection, segmentation and control, and recently its applicability in neurosciences, especially in brain tissue segmentation, is also increasing $[34,42,43]$.

This research proposes a new computer vision method that identifies changes in parenchymal density in Non-Contrast Computer Tomography for the detection of early stages of ischemic stroke. Based on the analysis of cerebral symmetry, the method identifies non-pathological and cases with abnormalities. The identification of normal tissues (CSF, WM and GM), using fuzzy logic techniques, simplifies the detection of parenchymal changes of ischemic stroke.

\section{Methodology}

Figure 1 outlines the proposed methodology. Each step is then presented in the following sections.

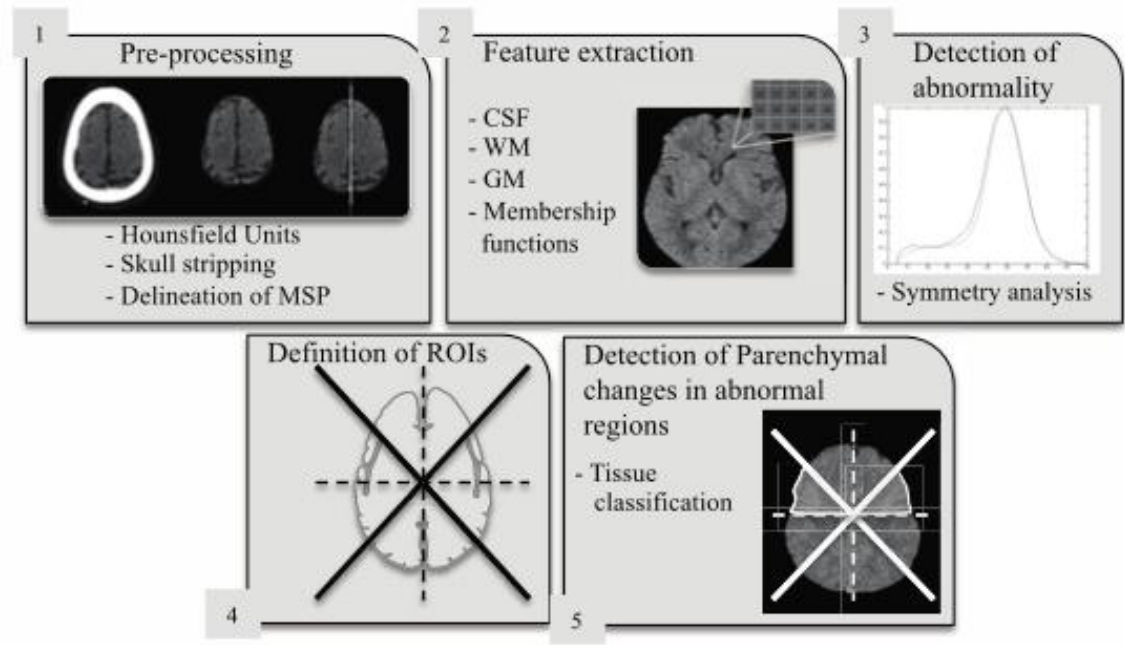


Fig. 1. General outline of the methodology.

\subsection{Pre-processing}

In this first stage of the methodology it was necessary to carry out the image standardization through three steps: the conversion of the gray scale to $\mathrm{HU}$, since it is the main characterization feature in clinical practice; then, the implementation of previously developed algorithms [38] for skull stripping and to define the ROI (the brain parenchyma), and finally the delimitation of the Mid Sagittal Plane (MSP) for the symmetry analysis.

\subsection{Feature extraction}

At this stage, subsets of non-pathological brain tissue (from normal subjects) were selected with the aim of analyzing the HU characteristics [44]. These subsets were extracted from specific anatomical regions where each tissue is denser. For WM, the samples were obtained from the region of the knee of the corpus callosum and semioval centers; for gray matter, of the head and body of the caudate nucleus; and the ventricles for CSF. The training set was composed of 640 tissue samples: 4 samples (sub images of at least $20 \times 20$ pixels) of each tissue using 4 slices ( 1 slice per sample) of 40 cases from 3 CT scanners. Cases were collected from two hospitals located in Cd. Juárez, México. Their use in this research was approved by a bioethics committee. The samples were validated under the guidance of two expert neuroradiologists (7 years of experience) and according to the literature $[1,4]$.

Table 1. Values in Hounsfield Units of brain tissues in different slice thicknesses (in mm).

\begin{tabular}{ccccc}
\hline Tissue & thickness & $\min$ & $\max$ & avg \\
\hline \multirow{2}{*}{ CSF } & 1 & 0 & 14 & 6.43 \\
& 1.5 & 0 & 14 & 6.13 \\
& 3 & 0 & 14 & 5.28 \\
WM & 1 & 17 & 46 & 29.39 \\
& 1.5 & 20 & 36 & 29.85 \\
& 3 & 20 & 35 & 29.46 \\
GM & 1 & 28 & 64 & 46.43 \\
& 1.5 & 31 & 59 & 44.54 \\
& 3 & 33 & 57 & 43.8 \\
\hline
\end{tabular}

The training set was arranged into three different vectors - CSF, WM and GM- and their corresponding histograms were obtained (Figure 2). A membership function associated for each of the tissues, $\mu \mathrm{CSF}, \mu \mathrm{WM}$ and $\mu \mathrm{GM}$, was implemented using Equation (1). 


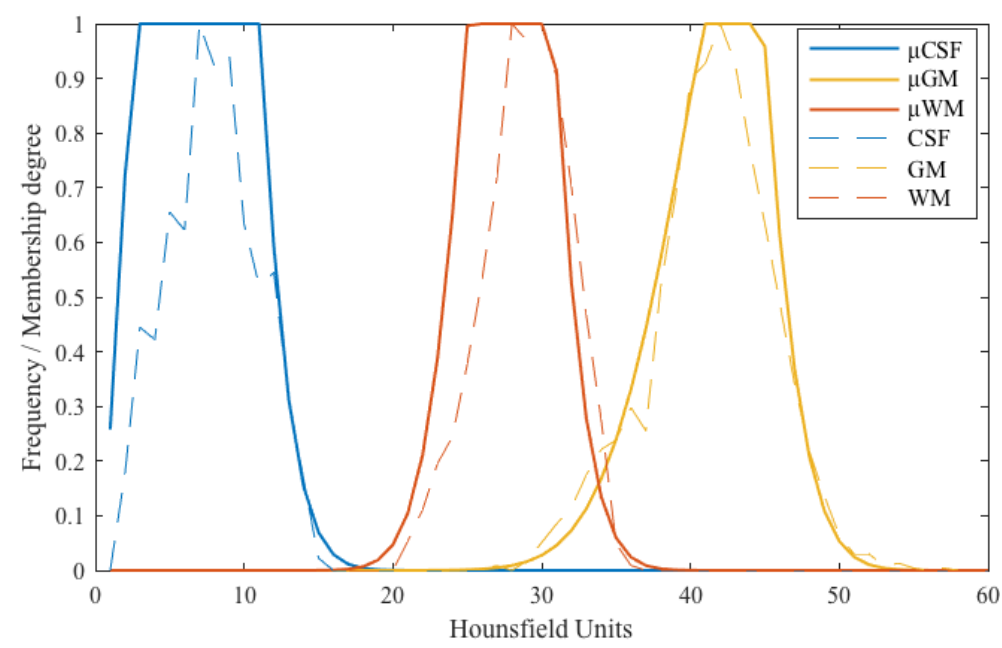

Fig. 2. Histograms of CSF, WM and GM image training set $1.5 \mathrm{~mm}$ thickness, and their corresponding membership functions.

$$
\mu_{C}\left(c_{i j}\right)=\min \left\{1, \frac{\operatorname{Lcdf}\left(c_{i j}\right)}{a_{1}}, \frac{R c d f\left(c_{i j}\right)}{a_{2}}\right\}
$$

where $C$ is the characteristic to be evaluated in population $P . c_{i j}$ is the value taken by $C$ when it is evaluated in pixel $p_{i j}$. $L c d f\left(c_{i j}\right)$ and $\operatorname{Rcd} f\left(c_{i j}\right)$ are the values of the left and right cumulative distribution functions $(c d f s)$ associated with the pixel $p_{i j}$ for the characteristic $C . a_{1}$ and $a_{2}$ are parameters that must be adjusted according to the overlap between the populations to be evaluated, increasing their value proportionally to their overlap. The advantage of considering the $c d f s$ is twofold: on the one hand, they can be used to better cover the data, especially in the case of asymmetric distributions; and on the other hand, provide better classification results when the histograms of datasets overlap $[45,46]$.

\subsection{Detection of abnormality}

The analysis of brain symmetry to detect abnormalities has been the basis of several works in the literature. It is a rapid method that locates a region of abnormality. The method takes advantage of the fact that a normal brain structure is quasi-symmetrical (Figure 3a), the hemispheres are divided by an axis of symmetry and abnormalities typically disrupt this symmetry (Figure $3 \mathrm{~b}$ ). 

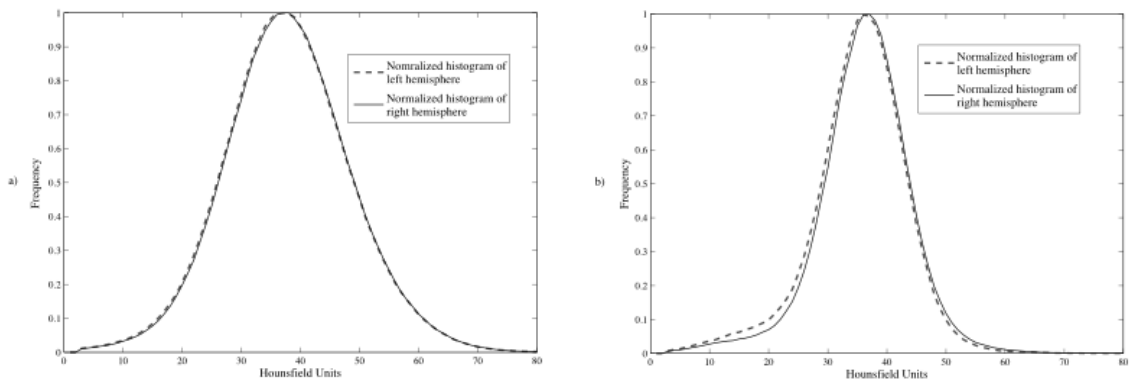

Fig. 3. a) Normalized histograms of left and right hemispheres of a non-pathological case. b). Normalized histograms of left and right hemispheres of a stroke case.

The analysis was performed using Jeffrey's divergence coefficient according to Equation (2). Divergence is a weaker notion than distance in mathematics. From the different divergence measures in the literature, perhaps the most prominent includes the Kullback-Leibler divergence (KLD). A big inconvenience of the KLD, especially in the context of practical applications, is its non-symmetricity [47]. Jeffrey's divergence (JD) is a simple way of making KLD symmetric; it is numerically stable and robust with respect to noise and the size of histogram bins [48]. JD and KLD are an empirical measure of the distributions similarity based on their relative entropy. Given two histograms $P$ and $Q$, with $p_{i}$ and $q_{i}$ denoting the histogram entries, the Jeffrey divergence is defined as:

$$
d_{J}(P, Q)=\sum_{i}\left\{p_{i} \log \frac{p_{i}}{m_{i}}+q_{i} \log \frac{q_{i}}{m_{i}}\right\}
$$

where $m_{i}=\frac{p_{i}+q_{i}}{2}$. P and Q are the histograms of HU data of the left and right hemispheres, respectively. The histograms are normalized so that the analysis is performed using the histogram signature and notwithstanding the number of pixels in each tissue. This allows diminishing the effect of the property of quasi-symmetry of the brain.

We analyzed 40 non-pathological training cases where it was observed that regardless the type of tissue (represented by the distribution of the histograms in HU), no case presented a divergence greater than 0.35 (Figure 4). A threshold $t=0.5$ could be established according to the maximum divergence of all cases, $\mathrm{m}$, plus twice the standard deviation of the maximum divergences of the cases. We denote the abnormality of a case $\mathrm{C}$ according to:

$$
C=\left\{\begin{array}{cc}
\text { abnormal } & \text { if } \max \left(d_{J}\right)>t \\
\text { normal } & \text { otherwise }
\end{array}\right\}
$$




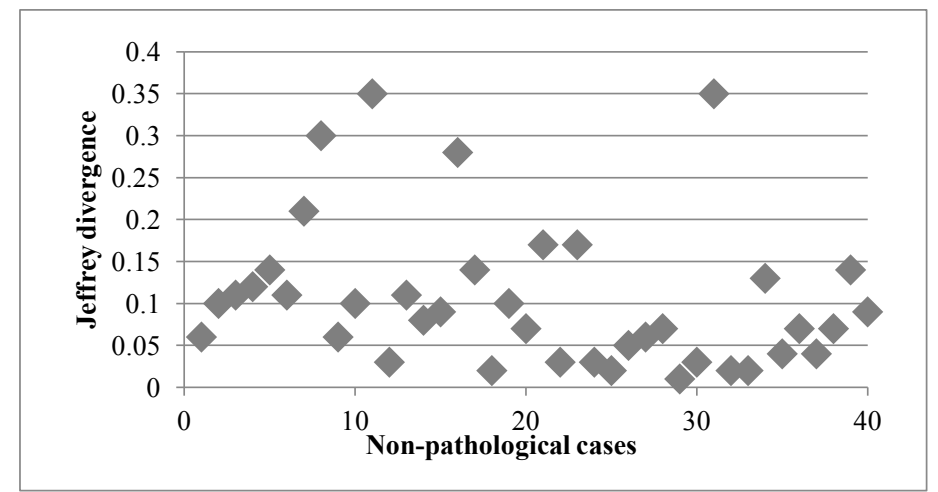

Fig. 4. Jeffrey divergence of hemispheres in non-pathological cases.

\subsection{Definition of ROIs}

All cases that present abnormalities are taken to a second level of classification by a contralateral analysis of brain regions. The brain volume is divided into 8 regions (Figure 5) by 4 edges that cross the entire volume through the center (recalling that the brain was aligned to the center and the patient's rotation angles were corrected in the preprocessing stage).

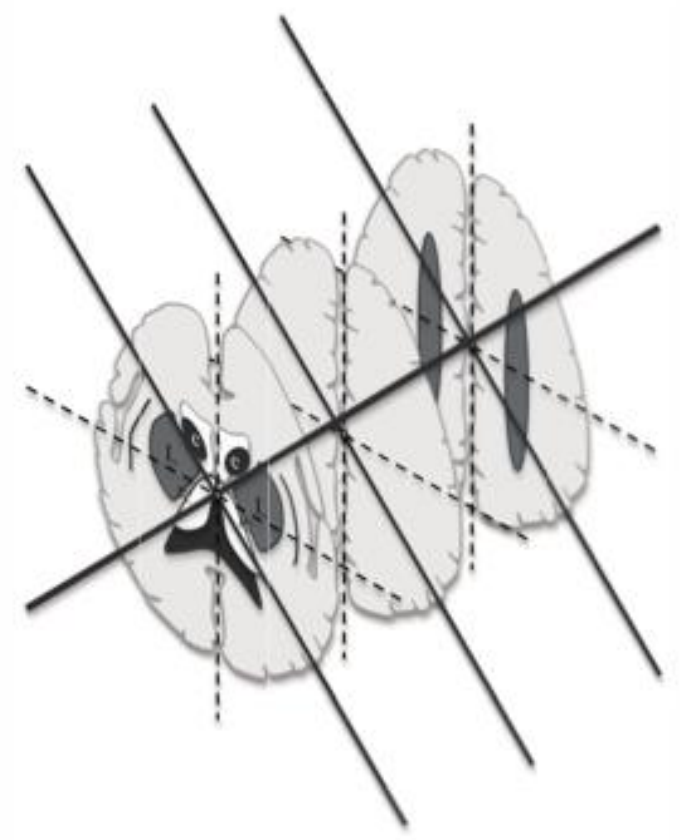

Fig. 5. Proposed delimitation of brain regions for contralateral analysis. 


\subsection{Detection of parenchymal changes in abnormal regions}

Once the regions are defined, each region of one hemisphere is evaluated against its counterpart on the other hemisphere. The symmetry analysis is performed again using the Jeffrey divergence in order to identify abnormalities (Equation 1). If the condition of Equation 3 is fulfilled, all voxels within the abnormal region are analyzed.

The analysis is feasible because normal brain tissues were characterized by membership functions in HU. A voxel $v_{i j}$ is considered WM if $\mu W M\left(v_{i j}\right)>\mu C S F\left(v_{i j}\right)$ and $\mu W M\left(v_{i j}\right)>\mu G M\left(v_{i j}\right)$. A voxel is GM if $\mu G M\left(v_{i j}\right)>$ $\mu \operatorname{CSF}\left(v_{i j}\right)$ and $\mu G M\left(v_{i j}\right)>\mu W M\left(v_{i j}\right)$. Finally, a voxel is CSF if $\mu C S F\left(v_{i j}\right)>\mu W M\left(v_{i j}\right)$ and $\mu C S F\left(v_{i j}\right)>\mu G M$ $\left(v_{i j}\right)$. The classification will result in a membership degree with values between 0 and 1 and will be represented visually in a range of 256 gray levels, as shown in Figure 6.

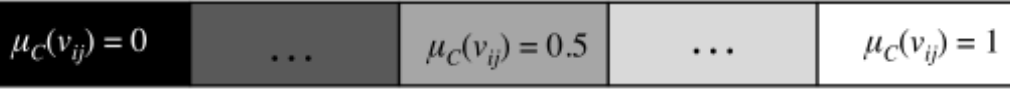

Fig. 6. Palette of gray levels representing the classification membership degrees.

\section{Results}

The following sections present and discuss the results of each step of the proposed methodology.

\subsection{Feature extraction in $H U$}

The extracted characteristics were evaluated by the quantitative classification of synthetic images generated from real tissues. Tissue segments were obtained from cases that were not used in the training phase. Figure 7 shows two examples of synthetic images and their corresponding classification using black for $\mathrm{CSF}$, gray for GM and white for WM. 

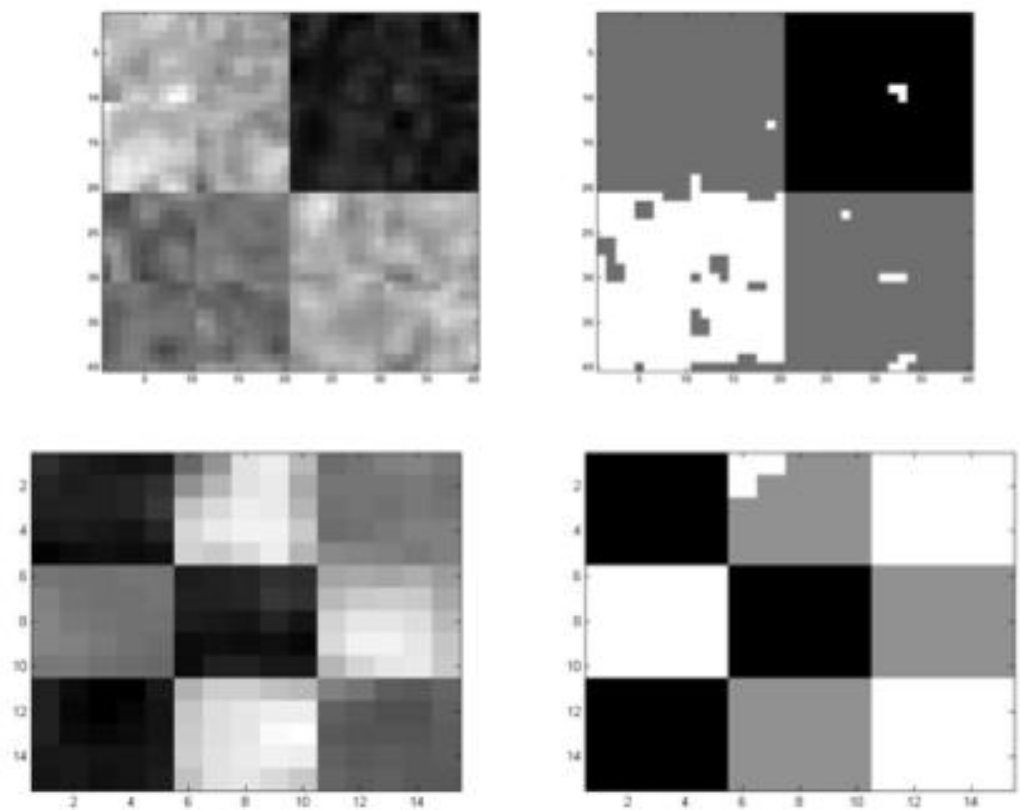

Fig. 7. Examples of synthetic images (left) and their classification (right).

Figure 8 contains the results of TPR and SPC measurements of the 27 synthetic images generated with a mean of 95 and 94, respectively. Some misclassifications were presented in the HU, in which the WM overlaps with GM (this overlap can be easily observed in the histograms of Figure 2).
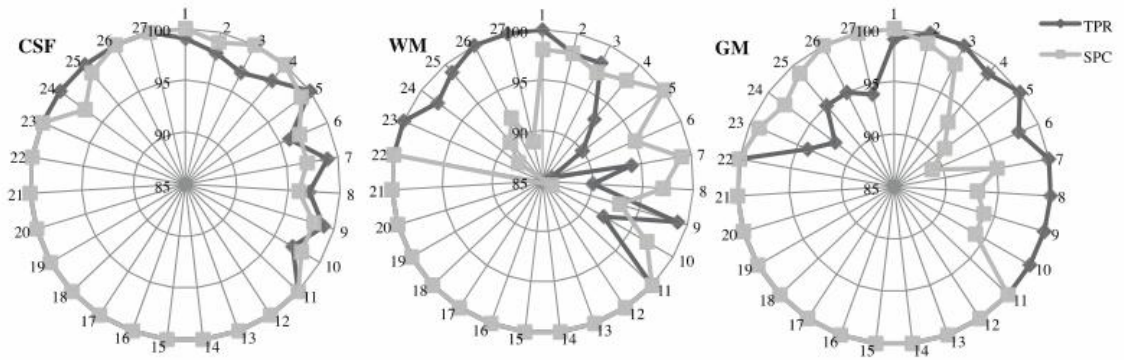

Fig. 8. Results of classification of tissues in synthetic images. Some misclassifications were presented in the HU, in which the WM overlaps with GM.

\subsection{Detection of an abnormality}

In order to validate the detection, a set of tests conformed by 54 non-pathological cases (Figure 9) and 20 stroke cases was used. 
a)

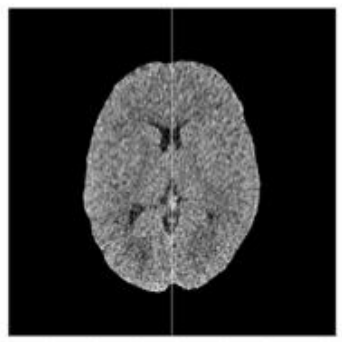

c)

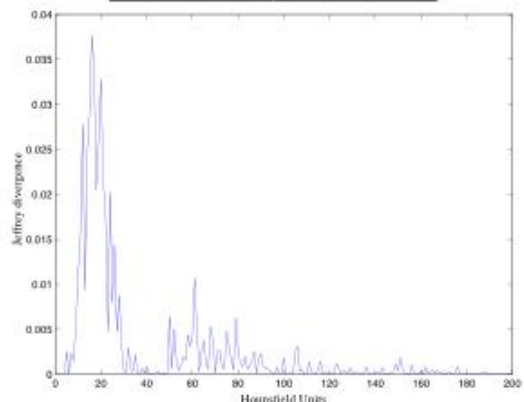

b)
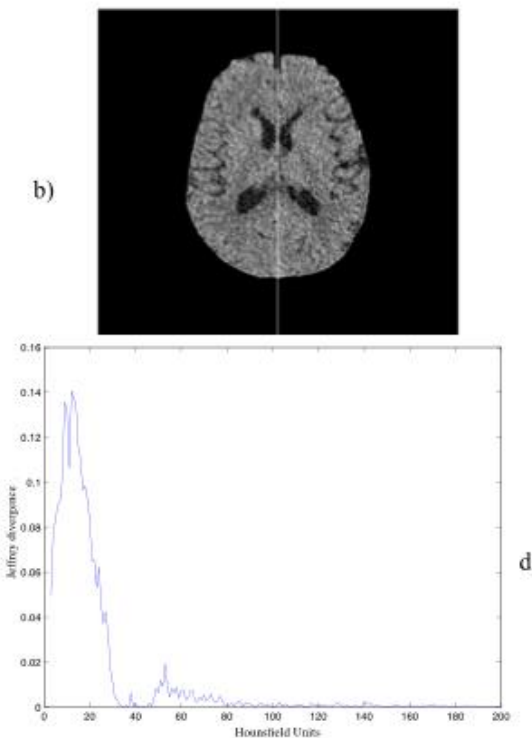

Fig. 9. Examples of non-pathological cases (a-b) and their corresponding divergence measurements (c-d).

It can be observed that the divergence values are greater in the stroke cases (Figure 11), exceeding the threshold $t$, than in non-pathological cases (Figure 10). It is important to emphasize that the analyzed pathological cases are set in different stages: chronic, sub-acute, acute and hyperacute. The evaluation of these experiments resulted in a sensitivity of $91 \%$ and specificity of $100 \%$.

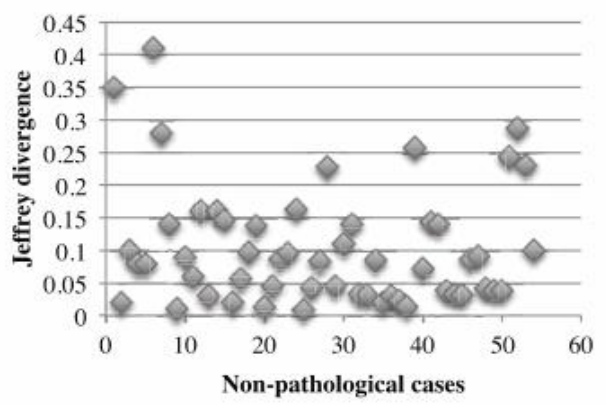

Fig. 10. Detection of abnormality of test cases: non-pathological patients. All cases are below threshold $t$. 


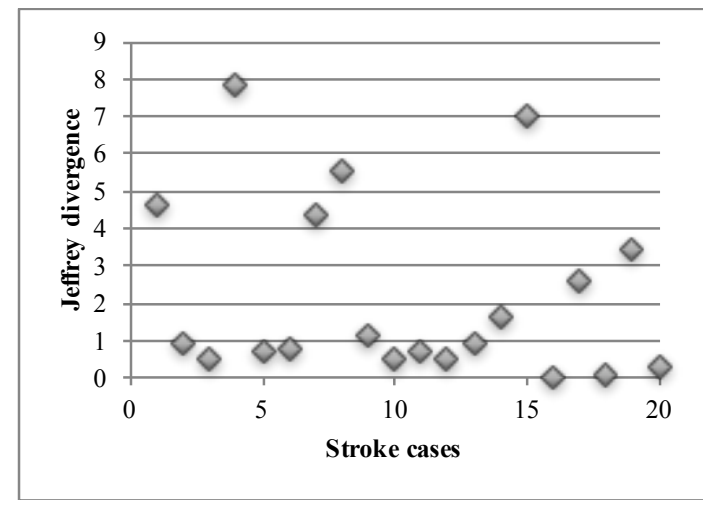

Fig. 11. Detection of abnormality of test cases: stroke patients.

\subsection{Detection of parenchymal changes in abnormal regions}

After discarding all the cases identified as normal, we proceeded to evaluate the different regions of interest proposed in this study, measuring 4 divergences per case. Table 2 presents the stage and location of the stroke, the divergence values in the four regions, and the overall predictive value, or diagnostic efficiency.

Table 2. Stage and location of the stroke, divergence values, and overall accuracy of abnormal cases.

\begin{tabular}{|c|c|c|c|c|c|c|c|}
\hline \multirow[b]{2}{*}{$\#$} & \multirow[b]{2}{*}{ Stage } & \multicolumn{6}{|c|}{ max Jdiv per region } \\
\hline & & Location & 1 & 2 & 3 & 4 & ACC \\
\hline \multirow{2}{*}{1} & \multirow{2}{*}{ sub } & \multirow{2}{*}{$\begin{array}{l}\text { Genu and posterior limb of } \\
\text { the left internal capsule }\end{array}$} & 7.81 & 6.32 & 8.9 & 4.35 & \multirow{2}{*}{100} \\
\hline & & & TP & $\mathrm{TP}$ & $\mathrm{TP}$ & TP & \\
\hline \multirow{2}{*}{2} & \multirow{2}{*}{ sub } & \multirow{2}{*}{$\begin{array}{l}\text { Genu and posterior limb of } \\
\text { the left internal capsule }\end{array}$} & 11.89 & 0.55 & 17 & 3.81 & \multirow{2}{*}{100} \\
\hline & & & TP & $\mathrm{TP}$ & $\mathrm{TP}$ & ТP & \\
\hline \multirow{2}{*}{3} & \multirow{2}{*}{ sub } & \multirow{2}{*}{ Left MCA territory } & 1.32 & 4.06 & 4.06 & 4.48 & \multirow{2}{*}{100} \\
\hline & & & TP & $\mathrm{TP}$ & $\mathrm{TP}$ & ТР & \\
\hline \multirow{2}{*}{4} & \multirow{2}{*}{ sub } & \multirow{2}{*}{$\begin{array}{l}\text { Posterior limb of the left } \\
\text { internal capsule }\end{array}$} & 4.76 & 0.29 & 0.12 & 0.55 & \multirow{2}{*}{100} \\
\hline & & & TP & $\mathrm{TN}$ & $\mathrm{TN}$ & $\mathrm{TN}$ & \\
\hline \multirow{2}{*}{5} & \multirow{2}{*}{ sub } & \multirow{2}{*}{$\begin{array}{l}\text { Ventral surface of the left } \\
\text { frontal lobe }\end{array}$} & 0.64 & 3.18 & 1.19 & 0.24 & \multirow{2}{*}{100} \\
\hline & & & ТP & TP & $\mathrm{TP}$ & $\mathrm{TN}$ & \\
\hline \multirow{2}{*}{6} & \multirow{2}{*}{ chro } & \multirow{2}{*}{$\begin{array}{l}\text { Posterior limb of the left } \\
\text { internal capsule }\end{array}$} & 0.49 & 5.79 & 0.5 & 0.37 & \multirow{2}{*}{100} \\
\hline & & & $\mathrm{TN}$ & $\mathrm{TP}$ & $\mathrm{TN}$ & $\mathrm{TN}$ & \\
\hline \multirow{2}{*}{7} & \multirow{2}{*}{ sub } & \multirow{2}{*}{ MCA and PCA territory } & 0.93 & 0.61 & 4.77 & 0.47 & \multirow{2}{*}{100} \\
\hline & & & $\mathrm{TP}$ & $\mathrm{TP}$ & TP & $\mathrm{TN}$ & \\
\hline 8 & sub & Bilateral occipital PCA & 9.58 & 3.20 & 9.13 & 5.50 & 100 \\
\hline
\end{tabular}




\begin{tabular}{|c|c|c|c|c|c|c|c|}
\hline & & territory & ТP & TP & ТP & $\mathrm{TP}$ & \\
\hline \multirow{2}{*}{9} & \multirow{2}{*}{ chro } & \multirow{2}{*}{ Left frontal recess } & 0.16 & 0.02 & 0.04 & 0.00 & \multirow{2}{*}{80} \\
\hline & & & $\mathrm{TN}$ & $\mathrm{FN}$ & $\mathrm{TN}$ & $\mathrm{TN}$ & \\
\hline \multirow{2}{*}{10} & \multirow{2}{*}{ sub } & \multirow{2}{*}{$\begin{array}{l}\text { Lateral aspect of the left } \\
\text { cerebral hemisphere }\end{array}$} & 7.92 & 9.56 & 3.42 & 0.70 & \multirow{2}{*}{10} \\
\hline & & & $\mathrm{TP}$ & $\mathrm{TP}$ & $\mathrm{TP}$ & $\mathrm{TP}$ & \\
\hline \multirow{2}{*}{11} & \multirow{2}{*}{ sub } & \multirow{2}{*}{ Left paracentral lobule } & 0.51 & 0.26 & 0.47 & 0.20 & \multirow{2}{*}{100} \\
\hline & & & $\mathrm{TP}$ & $\mathrm{TN}$ & $\mathrm{TN}$ & $\mathrm{TN}$ & \\
\hline \multirow{2}{*}{12} & \multirow{2}{*}{ chro } & \multirow{2}{*}{$\begin{array}{l}\text { Posterior limb of the left } \\
\text { internal capsule }\end{array}$} & 8.80 & 5.11 & 6.69 & 1.51 & \multirow{2}{*}{100} \\
\hline & & & $\mathrm{TN}$ & $\mathrm{TP}$ & $\mathrm{TN}$ & $\mathrm{TN}$ & \\
\hline \multirow{2}{*}{13} & \multirow{2}{*}{ sub } & \multirow{2}{*}{ Supra / infratentorial } & 0.08 & 0.14 & 1.29 & 1.36 & \multirow{2}{*}{100} \\
\hline & & & $\mathrm{TN}$ & $\mathrm{TN}$ & $\mathrm{TP}$ & $\mathrm{TP}$ & \\
\hline \multirow{2}{*}{14} & \multirow{2}{*}{ acute } & \multirow{2}{*}{ Left centrum semiovale } & 4.65 & 1.28 & 3.91 & 1.02 & \multirow{2}{*}{100} \\
\hline & & & $\mathrm{TP}$ & $\mathrm{TP}$ & ТP & ТP & \\
\hline \multirow{2}{*}{15} & \multirow{2}{*}{ hyper } & \multirow{2}{*}{ Cortico subcortical } & 0.5 & 0.38 & 0.83 & 1.31 & \multirow{2}{*}{100} \\
\hline & & & $\mathrm{TN}$ & $\mathrm{TN}$ & $\mathrm{TP}$ & $\mathrm{TP}$ & \\
\hline \multirow{2}{*}{16} & \multirow{2}{*}{ acute } & \multirow{2}{*}{ Right MCA territory } & 1.23 & 0.45 & 1.24 & 0.22 & \multirow{2}{*}{80} \\
\hline & & & $\mathrm{TP}$ & $\mathrm{FN}$ & $\mathrm{TP}$ & $\mathrm{TN}$ & \\
\hline \multirow{2}{*}{17} & hyner & Ventral surface of left & 1 & 0.14 & 0.19 & 1.46 & 100 \\
\hline & пурег & occipital and temporal lobe & $\mathrm{TP}$ & $\mathrm{TN}$ & $\mathrm{TN}$ & $\mathrm{TP}$ & \\
\hline 18 & hyner & Left olobus nallidus & 0.77 & 0.24 & 1.04 & 0.17 & 100 \\
\hline 10 & пурег & Left globus pantidus & $\mathrm{TP}$ & $\mathrm{TN}$ & $\mathrm{TP}$ & $\mathrm{TN}$ & 100 \\
\hline 10 & 00 & I oft porocontrol & 1.21 & 1.68 & 0.30 & 0.06 & 100 \\
\hline 19 & acule & Leit paracentral Iooure & $\mathrm{TP}$ & $\mathrm{TP}$ & $\mathrm{TN}$ & $\mathrm{TN}$ & 100 \\
\hline 20 & & Ventral surface of the left & 1.51 & 0.19 & 0.10 & 0.10 & \\
\hline & & & $\mathrm{TP}$ & $\mathrm{TN}$ & $\mathrm{TN}$ & $\mathrm{TN}$ & \\
\hline
\end{tabular}

In the twenty diagnosed cases, a total of 80 regions were evaluated, detecting abnormalities in 47 of 49 regions that were previously diagnosed as abnormal. In cases 9 and 16, in zone 2, the lesion appeared in less than $10 \%$ of the voxels, therefore this area was detected as normal. To avoid these false negatives, instead of carrying out the detection within geometrically defined regions, it will be necessary to consider regions delimited by the vascular territories, so that the lesion is not arbitrarily divided into multiple areas.

Figure 12 shows an example of the divergence measurements of the 4 contralateral regions. Once the regions in which divergence exceeds $t$ are detected (regions 2 and 3), the membership to CSF, WM and GM classes is evaluated on each voxel. Figure 13 illustrates the classification of one of the slices of zone 2 (a). The membership degrees of pixels to GM class on the left and right hemispheres are shown in (b.1) and (c.1), respectively; (b.2) and (c.2) to WM, and (b.3) and (c.3) to CSF. When comparing (b.1) and (c.1) a greater 
number of pixels classified to GM in the left hemisphere are observed (b.1). There should be a similar number of pixels classified as GM in (c.1), which may be indicative of tissue hypodensity in the right hemisphere. This provokes a greater number of pixels classified as WM in the right hemisphere (c.2).
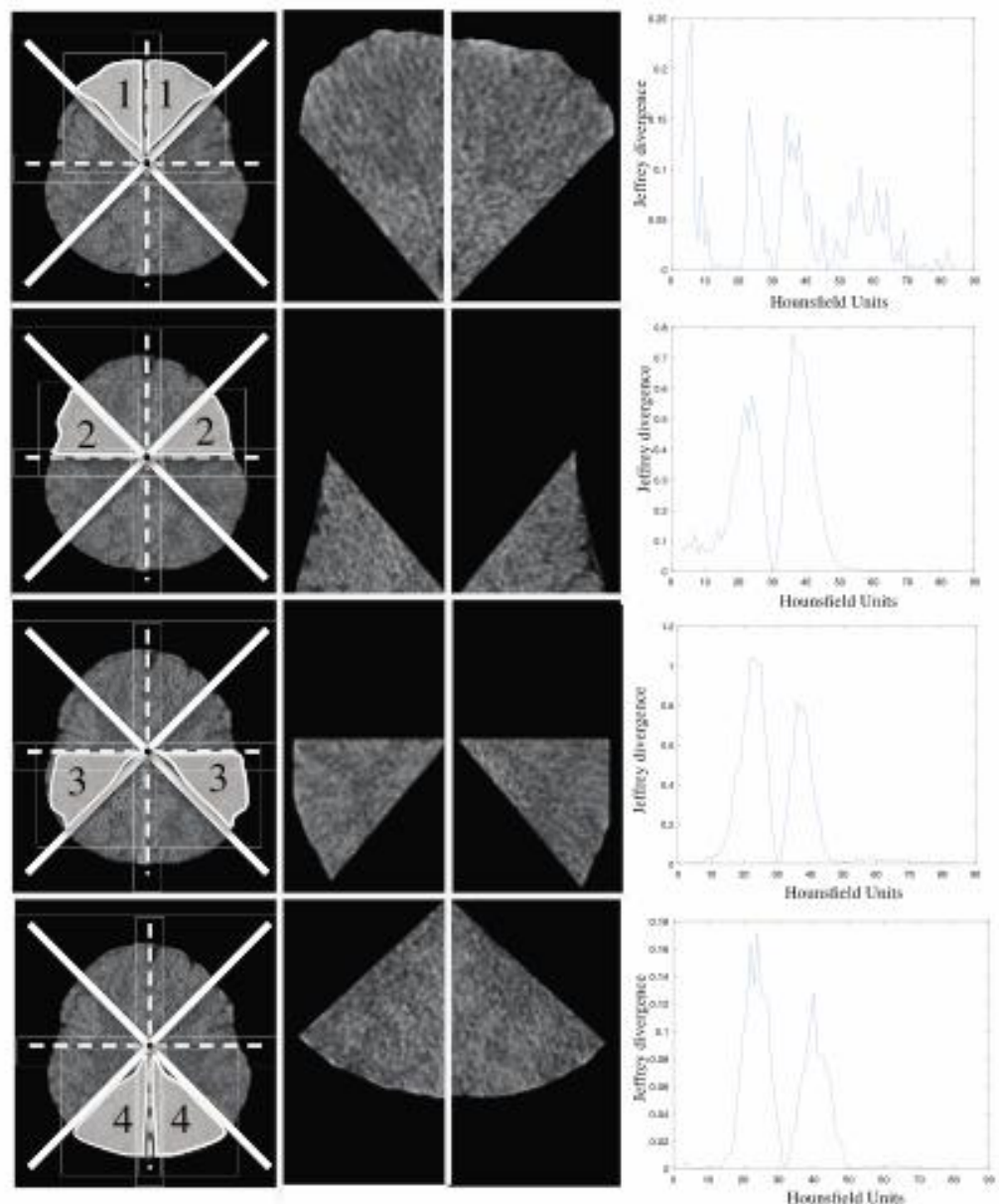

Fig. 12. Example of symmetry evaluation by regions in an abnormal case. 
(a)

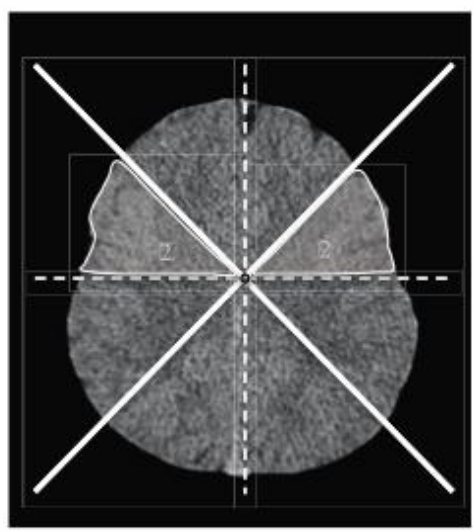

(b)
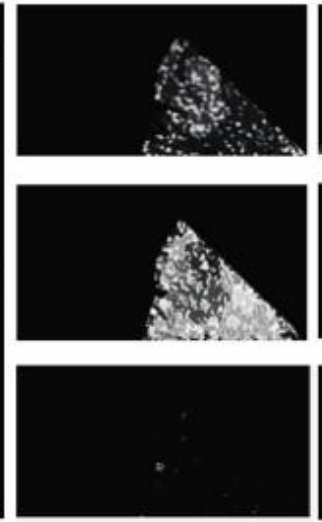

(c)
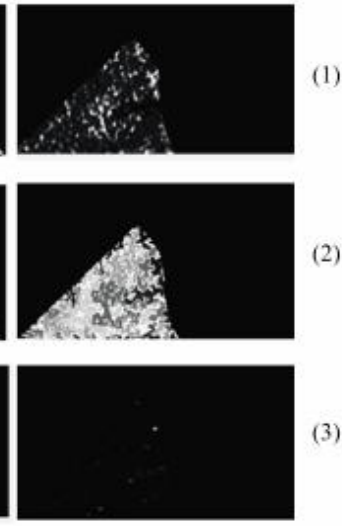

\section{1)}

2)

(3)

Fig. 13. a) Example of one of the slices used to classify the areas of interest. b) right hemisphere, c) left hemisphere. 1) Classification of GM. 2) WM. 3) CSF.

The case shown in Figures 12 and 13 was diagnosed as a hyperacute ischemic stroke. NCCT was acquired within the first three hours after the onset of the stroke. Therefore, the identification of the stroke was questionable, requiring a diffusion-weighted MRI. The study was performed two hours after NCCT, confirming the diagnosis (Figure 14). The abnormalities detected by the method occurred at the same location of the affected area in MRI. It is necessary to consider that the MRI area may be larger due to the higher MRI resolution to detect ischemic changes using diffusion and the time between the two studies.

(a)

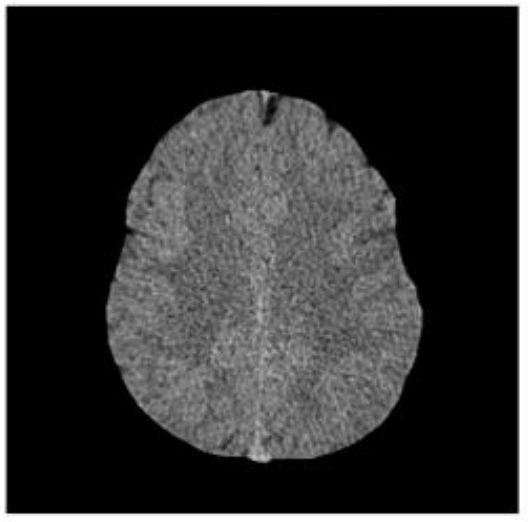

(b)

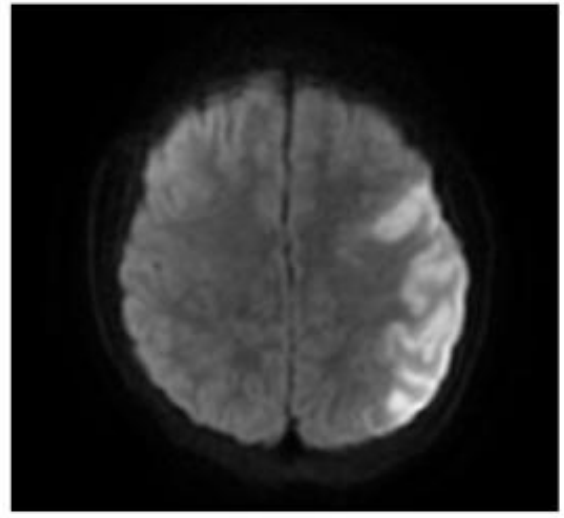

Fig. 14. Study by image for the detection of ischemic stroke of the same patient a) modality in NCCT, b) modality in diffusion weighted MRI two hours after the acquisition of the CT study. 


\section{Discussion}

The detection of an ischemic stroke is performed by identifying atypical values in regions in which healthy tissues are expected because it is difficult to characterize parenchymal changes of an ischemic stroke from a reduced sample of previously diagnosed cases, specially hyperacute cases that are generally not diagnosed. It is also important to consider that HU will vary according to the type and area of the stroke and the elapsed time. For example, it may be unchallenging to detect a chronic ischemic stroke regardless of the tissue in which it is located because its values in HU do not overlap with the HU of healthy tissues, so a membership function adjusted to that type of infarction is feasible but has minimal clinical relevance since these cases are easily diagnosed because their difference in density is remarkable. The problem of adjusting a membership function for cases of hyperacute ischemic stroke is that its levels in HU change according to the affected tissue, which means that, if it affects the GM, the detected pixels will tend to resemble WM. Because the diagnoses of specialists are variable regarding this type of stroke, it was considered helpful to the specialist to detect a region of interest and to identify the tissues separately in order to provide a better differentiation between hemispheres, which can lead to a timely diagnosis of ischemic stroke.

In addition to the histogram signatures where the expert can quantitatively verify the levels in HU in which the histogram changes, along with the divergence values, he will be able to perform a qualitative analysis in the resulting images of the classification to verify if there is indeed a significant disparity between the contralateral regions, and thus be able to observe changes between the tissues more easily. The expert, having this information, could determine if it is either an ischemic stroke, another pathology, or a false positive. In this way, the method can reduce the search area to accelerate the diagnosis and initiate a more timely treatment, or to discard the presence of parenchymal changes of ischemic stroke.

\section{Conclusions}

The main objective of this article was to provide a new automated, early detection of parenchymal changes of ischemic stroke in NCCT images. The abnormality was detected according to a symmetry analysis between the left and right hemispheres of the brain. The hemispheres of training cases were compared using the Jeffrey divergence, establishing a threshold to determine abnormality when it was exceeded. In our analysis focused on real images, our approach demonstrated the ability to correctly classify all the cases that were evaluated as can be inferred from its excellent sensitivity and specificity.

The different abnormal regions were evaluated and the presence of parenchymal changes of ischemic stroke was detected by the identification of tissues using fuzzy logic techniques. In our approach, the design of the membership functions took the advantage of using samples of non-pathological tissues (CSF, WM, and $\mathrm{GM}$ ) that allowed their characterization and adjustment. We obtained a very high performance (an average sensitivity of $95 \%$ and a specificity of $94 \%$ ) in the quantitative evaluation using real tissue images.

Based on the obtained results, this new method can be extended to a CADe that could reduce detection time, since the non-pathological cases are discarded. Because of abnormal regions are identified, the expert can prioritize the examination to a previously delimited region, decreasing the diagnostic time. The identification of tissues allows a better visualization of the region to be evaluated, helping to discard or confirm a stroke. 
Finally, the excellent results showed that the proposed approach, could be extended to the analysis of other pathologies or to be applied jointly with cerebral atlases in order to help in the delineation of the different cerebral regions.

\section{Acknowledgments}

All procedures performed in studies involving human participants were in accordance with the ethical standards of the institutional and/or national research committee and with the 1964 Helsinki declaration and its later amendments or comparable ethical standards.

Informed consent was obtained from all individual participants included in the study.

A. Davis received partial economic support from National Council of Science and Technology of Mexico (Grant number: 580575). N. Gordillo, E. Montseny, F. X. Aymerich, M. López-Córdova, L. Ortega-Maynez and B. Mederos-Madrazo declare that they have no conflict of interest.

\section{References}

[1] J.P. Mohr, P.A. Wolf, J.C. Grotta, M.A. Moskowitz, M.R. Mayberg, R. Von Kummer, Stroke: pathophysiology, diagnosis, and management, Elsevier saunders, Philadelphia, 2011.

[2] Stroke Information Page | National Institute of Neurological Disorders and Stroke, (n.d.). https://www.ninds.nih.gov/Disorders/All-Disorders/Stroke-Information-Page (accessed March 21, 2017).

[3] J.L. Saver, Time Is Brain--Quantified, Stroke. 37 (2006) 263-266. doi:10.1161/01.STR.0000196957.55928.ab.

[4] M. Castillo, Neurorradiología, E. journal, Buenos Aires, 2004.

[5] M.T. Torbey, M.H. Selim, The Stroke Book, second edi, Cambridge University Press, New York, 2013.

[6] J.M. Wardlaw, O. Mielke, Early signs of brain infarction at CT: observer reliability and outcome after thrombolytic treatment-systematic review., Radiology. 235 (2005) 444-453. doi:10.1148/radiol.2352040262.

[7] M.P. Marks, E.B. Holmgren, A.J. Fox, S. Patel, R. von Kummer, J. Froehlich, Evaluation of early computed tomographic findings in acute ischemic stroke., Stroke. 30 (1999) 389-92. http://www.ncbi.nlm.nih.gov/pubmed/9933276 (accessed April 2, 2016).

[8] C.L. Truwit, A.J. Barkovich, A. Gean-Marton, N. Hibri, D. Norman, Loss of the insular ribbon: another early CT sign of acute middle cerebral artery infarction., Radiology. 176 (1990) 801-6. doi:10.1148/radiology.176.3.2389039.

[9] R. von Kummer, U. Meyding-Lamadé, M. Forsting, L. Rosin, K. Rieke, W. Hacke, K. Sartor, Sensitivity and prognostic value of early CT in occlusion of the middle cerebral artery trunk., AJNR. Am. J. Neuroradiol. 15 (1994) 9-15-8. http://www.ncbi.nlm.nih.gov/pubmed/8141071 (accessed March 2, 2016).

[10] E.F. Wijdicks, M.N. Diringer, Middle cerebral artery territory infarction and early brain swelling: progression and effect of age on outcome., Mayo Clin. Proc. 73 (1998) 829-36. doi:10.4065/73.9.829.

[11] I. Dzialowski, E. Klotz, S. Goericke, A. Doerfler, M. Forsting, R. von Kummer, Ischemic brain tissue water content: CT monitoring during middle cerebral artery occlusion and reperfusion in rats., Radiology. 243 (2007) 720-6. doi:10.1148/radiol.2432060137.

[12] R. von Kummer, J. Weber, Brain and vascular imaging in acute ischemic stroke: the potential of computed tomography., Neurology. 49 (1997) S52-5. http://www.ncbi.nlm.nih.gov/pubmed/9371151 (accessed December 12, 2016).

[13] M. Bendszus, H. Urbach, B. Meyer, R. Schultheiss, Improved CT diagnosis of acute middle cerebral artery territory infarcts with density-difference analysis, Neuroradiology. 39 (1997) 127-131.

[14] Office for official publications of the european Communities, European guidelines in quality criteria for computed tomography, Brussels, 1999.

[15] L. Bozzao, S. Bastianello, Correlation of angiographic and sequential CT findings in patients with evolving cerebral infaction, AJNR. Am. J. Neuroradiol. 10 (1989) 1215-1222.

[16] S.H. Horowitz, J.L. Zito, R. Donnarumma, M. Patel, J. Alvir, Computed Tomographic-Angiographic Findings Within the First Five Hours of Cerebral Infarction, (1990).

[17] W. Hacke, M. Kaste, C. Fieschi, D. Toni, E. Lesaffre, R. von Kummer, G. Boysen, E. Bluhmki, G. Höxter, M.H. Mahagne, Intravenous thrombolysis with recombinant tissue plasminogen activator for acute hemispheric stroke. The European 
Cooperative Acute Stroke Study (ECASS), JAMA. 274 (1995) 1017-25. http://www.ncbi.nlm.nih.gov/pubmed/7563451 (accessed December 12, 2016)

[18] J.C. Grotta, D. Chiu, M. Lu, S. Patel, S.R. Levine, B.C. Tilley, T.G. Brott, E.C. Haley, P.D. Lyden, R. Kothari, M. Frankel, C.A. Lewandowski, R. Libman, T. Kwiatkowski, J.P. Broderick, J.R. Marler, J. Corrigan, S. Huff, P. Mitsias, S. Talati, D. Tanne, Agreement and variability in the interpretation of early CT changes in stroke patients qualifying for intravenous rtPA therapy., Stroke. 30 (1999) 1528-1533. doi:10.1161/01.STR.30.8.1528.

[19] A. Przelaskowski, K. Sklinda, P. Bargie??, J. Walecki, M. Biesiadko-Matuszewska, M. Kazubek, Improved early stroke detection: Wavelet-based perception enhancement of computerized tomography exams, Comput. Biol. Med. 37 (2007) 524 533. doi:10.1016/j.compbiomed.2006.08.004.

[20] Fuk-hay Tang, Douglas K. S. Ng, Daniel H. K. Chow, An image feature approach for computer-aided detection of ischemic stroke, Comput. Biol. Med. 41 (2011) 529-536.

[21] J.L. Saver, M. Goyal, A. van der Lugt, B.K. Menon, C.B.L.M. Majoie, D.W. Dippel, B.C. Campbell, R.G. Nogueira, A.M. Demchuk, A. Tomasello, P. Cardona, T.G. Devlin, D.F. Frei, R. du Mesnil de Rochemont, O.A. Berkhemer, T.G. Jovin, A.H. Siddiqui, W.H. van Zwam, S.M. Davis, C. Castaño, B.L. Sapkota, P.S. Fransen, C. Molina, R.J. van Oostenbrugge, Á. Chamorro, H. Lingsma, F.L. Silver, G.A. Donnan, A. Shuaib, S. Brown, B. Stouch, P.J. Mitchell, A. Davalos, Y.B.W.E.M. Roos, M.D. Hill, C. Castano, B.L. Sapkota, P.S. Fransen, C. Molina, R.J. van Oostenbrugge, A. Chamorro, H. Lingsma, F.L. Silver, G.A. Donnan, A. Shuaib, S. Brown, B. Stouch, P.J. Mitchell, A. Davalos, Y.B.W.E.M. Roos, M.D. Hill, H. Collaborators, R.D. de Rochemont, O.A. Berkhemer, T.G. Jovin, A.H. Siddiqui, W.H. van Zwam, S.M. Davis, C. Castao, B.L. Sapkota, P.S. Fransen, C. Molina, R.J. van Oostenbrugge, A. Chamorro, H. Lingsma, F.L. Silver, G.A. Donnan, A. Shuaib, S. Brown, B. Stouch, P.J. Mitchell, A. Davalos, B.W.E.M. Roos, M.D. Hill, H. Collaborators, Time to Treatment With Endovascular Thrombectomy and Outcomes From Ischemic Stroke: A Meta-analysis, Jama. 316 (2016) 1279. doi:10.1001/jama.2016.13647.

[22] M. Chawla, S. Sharma, J. Sivaswamy, L. Kishore., A method for automatic detection and classification of stroke from brain CT images, in: 31st Annu. Int. Conf. IEEE EMBS, Estados Unidos, 2009.

[23] J.A. Maldjian, J. Chalela, S.E. Kasner, D. Liebeskind, J.A. Detre, Automated CT segmentation and analysis for acute middle cerebral artery stroke, Am. J. Neuroradiol. 22 (2001) 1050-1055.

[24] C. Grimm, Voxel-based CT analysis for improved detection of early CT signs in cerebral infarction, (2005).

[25] Zeyun Yu, C. Bajaj, A fast and adaptive method for image contrast enhancement, in: 2004 Int. Conf. Image Process. 2004. ICIP '04., IEEE, n.d.: pp. 1001-1004. doi:10.1109/ICIP.2004.1419470.

[26] Joung-Youn Kim, Lee-Sup Kim, Seung-Ho Hwang, An advanced contrast enhancement using partially overlapped sub-block histogram equalization, IEEE Trans. Circuits Syst. Video Technol. 11 (2001) 475-484. doi:10.1109/76.915354.

[27] N. Bonnier, E. Simoncelli, LOCALLY ADAPTIVE MULTISCALE CONTRAST OPTIMIZATION, Image Process. Soc. Soc. I (2005) 949-952.

[28] D.. Hammond, E. Simoncelli, Nonlinear image representation via local multiscale orientation, 2005.

[29] L.M. Fayad, Y. Jin, A.F. Laine, Y.M. Berkmen, G.D. Pearson, B. Freedman, R. Van Heertum, Chest CT window settings with multiscale adaptive histogram equalization: pilot study., Radiology. 223 (2002) 845-52. doi:10.1148/radiol.2233010943.

[30] A.M. Boers, H.A. Marquering, J.J. Jochem, N.J. Besselink, O.A. Berkhemer, A. van der Lugt, L.F. Beenen, C.B. Majoie, Automated cerebral infarct volume measurement in follow-up noncontrast CT scans of patients with acute ischemic stroke., AJNR. Am. J. Neuroradiol. 34 (2013) 1522-7. doi:10.3174/ajnr.A3463.

[31] B.C. Stoel, H.A. Marquering, M. Staring, L.F.M. Beenen, C.H. Slump, Y.B. Roos, C.B.L.M. Majoie, Automated brain CT densitometry of early ischemic changes in acute stroke, J. Med. Imaging. 2 (2015) 1-11. doi:10.1117/1.JMI.2.1.014004.

[32] C. Herweh, P.A. Ringleb, G. Rauch, S. Gerry, L. Behrens, M. Mo, R. Gottorf, D. Richter, S. Schieber, S. Nagel, Performance of e-ASPECTS software in comparison to that of stroke physicians on assessing CT scans of acute ischemic stroke patients, Int. J. Stroke. 11 (2016) 438-445. doi:10.1177/1747493016632244.

[33] S. Nagel, D. Sinha, D. Day, W. Reith, R. Chapot, P. Papanagiotou, E.A. Warburton, P. Guyler, S. Tysoe, K. Fassbender, S. Walter, M. Essig, J. Heidenrich, A.A. Konstas, M. Harrison, M. Papadakis, E. Greveson, O. Joly, S. Gerry, H. Maguire, C. Roffe, J. Hampton-Till, A.M. Buchan, I.Q. Grunwald, e-ASPECTS software is non-inferior to neuroradiologists in applying the ASPECT score to computed tomography scans of acute ischemic stroke patients, Int. J. Stroke. 0 (2016) 1-8. doi:10.1177/1747493016681020.

[34] J. Ghasemi, R. Ghaderi, M.R. Karami Mollaei, S.A. Hojjatoleslami, A novel fuzzy Dempster-Shafer inference system for brain MRI segmentation, Inf. Sci. (Ny). 223 (2013) 205-220. doi:10.1016/j.ins.2012.08.026.

[35] A. Padma, R. Sukanesh, Segmentation and Classification of Brain CT Images Using Combined Wavelet Statistical Texture Features, Arab. J. Sci. Eng. 39 (2014) 767-776. doi:10.1007/s13369-013-0649-3.

[36] S. Valverde, A. Oliver, M. Cabezas, E. Roura, X. Lladó, Comparison of 10 brain tissue segmentation methods using revisited IBSR annotations, J. Magn. Reson. Imaging. 41 (2015) 93-101. doi:10.1002/jmri.24517.

[37] A. Demirhan, M. Toru, I. Guler, Segmentation of Tumor and Edema Along with Healthy Tissues of Brain Using Wavelets and Neural Networks, IEEE J. Biomed. Heal. Informatics. 19 (2015) 1451-1458. doi:10.1109/JBHI.2014.2360515.

[38] M. Kondo, K. Yamashita, T. Yoshiura, A. Hiwatash, T. Shirasaka, H. Arimura, Y. Nakamura, H. Honda, Histogram analysis with automated extraction of brain-tissue region from whole-brain CT images., Springerplus. 4 (2015) 788. doi:10.1186/s40064-015-1587-1.

[39] C. Aguilar, K. Edholm, A. Simmons, L. Cavallin, S. Muller, I. Skoog, E.M. Larsson, R. Axelsson, L.O. Wahlund, E. Westman, 
Automated CT-based segmentation and quantification of total intracranial volume, Eur. Radiol. 25 (2015) 3151-3160. doi:10.1007/s00330-015-3747-7.

[40] A. Patel, B. van Ginneken, F.J.A. Meijer, E.J. van Dijk, M. Prokop, R. Manniesing, Robust cranial cavity segmentation in CT and CT perfusion images of trauma and suspected stroke patients, Med. Image Anal. 36 (2017) 216-228. doi:10.1016/j.media.2016.12.002.

[41] L.A. Zadeh, Fuzzy logic — a personal perspective, 281 (2015) 4-20. doi:10.1016/j.fss.2015.05.009.

[42] G. Vishnuvarthanan, M.P. Rajasekaran, P. Subbaraj, A. Vishnuvarthanan, An unsupervised learning method with a clustering approach for tumor identification and tissue segmentation in magnetic resonance brain images, Appl. Soft Comput. J. 38 (2016) 190-212. doi:10.1016/j.asoc.2015.09.016.

[43] V. Borase, G. Naik, V. Londhe, Brain MR Image Segmentation for Tumor Detection using Artificial Neural Networks, Int. J. Eng. Comput. Sci. 6 (2017) 20160-20163. doi:10.18535/ijecs/v6i1.56.

[44] N. Gordillo-Castillo, A. Davis-Ortiz, F.X. Aymerich, J. Mejía-Muñoz, J. García-Quintero, M. López-Córdova, S. AndradeLuján, A fuzzy approach for feature extraction of brain tissues in Non-Contrast CT, Rev. Mex. Ing. Biomédica. 39 (2018) 112 120. doi:https://doi.org/10.17488/RMIB.39.1.10.

[45] N. Gordillo Castillo, E. Montseny, P. Sobrevilla, Contributions to Automatic and Unsupervised MRI Brain Tumor Segmentation: A New Fuzzy Approach, Technical University of Catalonia, 2010.

[46] N. Gordillo, E. Montseny, P. Sobrevilla, A new fuzzy approach to brain tumor segmentation, in: Int. Conf. Fuzzy Syst., Barcelona, Spain, 2010: pp. 1-8. doi:10.1109/FUZZY.2010.5584178.

[47] M. Budka, B. Gabrys, K. Musial, On accuracy of PDF divergence estimators and their applicability to representative data sampling, Entropy. 13 (2011) 1229-1266. doi:10.3390/e13071229.

[48] T.B.J.M. Puzicha Jan; Hofmann, Non-parametric Similarity Measures for Unsupervised Texture Segmentation and Image Retrieval, Comput. Vis. Pattern Recognition, 1997. Proceedings., 1997 IEEE Comput. Soc. Conf. (1997) 267-272. doi:10.1109/CVPR.1997.609331. 